\title{
Review of: "Individual and Environmental Risk Factors for COVID- 19 Mortality in Elderly"
}

\author{
Ly Luong $^{1}$ \\ 1 Hanoi University of Science
}

Potential competing interests: The author(s) declared that no potential competing interests exist.

That is an interesting study on a new issue of great interest today, the influence of individual and environmental factors on covid-19 mortality. As a reviewer, I have some minor comments for this study.

1. Please explain why the authors only use annual pollutant concentrations over the last two years of the study period as well as over the 20 days before the confirmed PCR diagnostic of COVID-19? Did you conduct any sensitivity analysis for a more prolonged exposure?

2. Could you please provide in the study the statistical description of pollutant concentrations over the last two year and over 20 days before patients undertook a PCR test?

3. Could you please provide (in 2.3. Statistical analyses) the formulas of models used in the study to make it easier for the reader to understand.

4. As I understand, annual pollutant concentrations over the last two years were used in the long-term environmental risk factors models. Please clarify this in 2.3.1.

5. The results section should be more detailed in the text.

6. I would like to see the same order of cities' names on the Y-axis in Figures 2 and 3, or the proportion of dead patients and average air pollutant concentration by the city can go together in one figure. 